\title{
Study of low weight percentage filler on dielectric properties of MWCNT-epoxy nanocomposites
}

\author{
Manindra Trihotri* ${ }^{\dagger}, \S$ U. K. Dwivedi ${ }^{\star}$, M. M. Malik*, \\ Fozia Haque Khan* and M. S. Qureshi* \\ *Department of Physics, Maulana Azad National Institute of Technology \\ Bhopal 462051 (MP), India \\ ${ }^{\dagger}$ Department of Physics, Laxmi Narain College of Technology \\ Bhopal 462021 (MP), India \\ Department of Physics, Amity University \\ Jaipur, Rajasthan \\ \$manindratrihotri@yahoo.com
}

Received 12 January 2016; Revised 13 June 2016; Accepted 6 August 2016; Published 26 September 2016

\begin{abstract}
An attempt is made to study the effect of low weight percentage multiwall carbon nanotube (MWCNT) powder on dielectric properties of MWCNT reinforced epoxy composites. For that MWCNT (of different low weight percentage) reinforced epoxy composite was prepared by dispersing the MWCNT in resin. Samples were prepared by solution casting process and characterized for their dielectric properties such as dielectric constant $\left(\varepsilon^{\prime}\right)$, dielectric dissipation factor $(\tan \delta)$ and AC conductivity $\left(\sigma_{\text {ac }}\right)$. The main objective is the investigation of the dielectric properties of the prepared samples at the low weight percentage of the filler at different temperatures and frequencies. From the two mechanisms of electrical conduction, first the leakage current obtained by the formation of a percolation network in the matrix and the other by tunneling of electrons formed among conductors nearby (tunneling current); here we are getting conduction by the second mechanism. Generally, leakage current makes more contribution to conductivity than tunneling current. Dielectric dissipation factor at $250 \mathrm{~Hz}$ frequency is greater than all other frequencies and starts increasing from $60^{\circ} \mathrm{C}$. The peak height of the transition temperature decreases with increasing frequency. This study shows that the addition of a low weight percentage of MWCNT can modify considerably the electrical behavior of epoxy nanocomposites without chemical functionalization of filler.
\end{abstract}

Keywords: Dielectric properties; tunneling current; MWCNT; epoxy; nanocomposites.

\section{Introduction}

Carbon nanotube (CNTs) is in focus nowadays due to their exceptional electrical, mechanical, and thermal properties. Reinforcement of small amounts of nanotubes in an insulating polymeric matrix found to render the electrical properties of the composite. The electrical properties of the polymer composites may vary from those of an insulating material to conductive composite with filler network depending on the concentration, property of the conducting fillers and dispersion of conducting fillers in polymeric matrix. ${ }^{1}$ Recently, CNTs are being very much investigated by the researchers globally with keen interest for the industrial applications. Since their discovery in 1991, CNTs have attracted enormous attention in research for their properties and their use in wide industrial applications. Single-wall nanotubes (SWNTs) were widely recognized in regard of their predicted properties among all types of CNTs. The remarkable properties of SWNTs are limited in usage because of their high cost and low yield of production. Multiwall carbon nanotubes (MWCNTs) are one of the most common and widely used nanofiller for enhancing the conductivity of the polymer composites.
MWCNT consists of multiple rolled layers (concentric tubes) of graphene with several concentrically aligned tubular graphene sheets, and the typical diameter in the range $8-30 \mathrm{~nm}^{2}$ MWCNTs can be used as reinforcing fillers for different components of composite materials with polymer, metal or ceramic matrices ${ }^{3}$ as chemical sensors by using laterally grown MWCNT, ${ }^{4,5}$ components of catalytic activity, ${ }^{6,7}$ and electromagnetic interference shielding materials ${ }^{8-10}$ for biomedical applications such as selective drug delivery. ${ }^{11,12}$ The most important applications of the CNTs are in the reinforcement of the different types of polymer (thermosetting and thermoplastic) matrix composites due to their high electrical, mechanical and extraordinary thermal conductivity.

The intrinsic potential of CNTs as reinforcing filler in elastomeric materials is very interesting. Despite a poor dispersion, small filler loading improves substantially the electrical and mechanical behavior of the soft matrix. Bokobza et al. ${ }^{13}$ showed that the high electrical conductivity of CNTfilled composites obtained at a relatively low volume fraction is one of the major attributes of CNTs since that allows retaining the desired mechanical properties. In polymer

This is an Open Access article published by World Scientific Publishing Company. It is distributed under the terms of the Creative Commons Attribution 4.0 (CC-BY) License. Further distribution of this work is permitted, provided the original work is properly cited. 
composites, dispersion of the used filler as well as interfacial interactions of the matrix and filler has been shown to be essential parameters for enhanced properties. The biggest challenge nowadays is to obtain a homogeneous dispersion of CNTs in a polymer matrix. This is because of the Vander Waals interactions between individual tubes which often lead to significant aggregation or agglomeration, and causes the reduction of the expected property improvements of the resulting composite. ${ }^{13}$ The conduction mechanism in CNT composites has been explained by considering that conductive path formed due to the CNT filler, causing the material to convert from an insulator to a conductor. These conductive paths are formed in the composite when the CNT concentration $\phi$ increases over a threshold value $\phi_{c}$. The dependency of the conductivity $\sigma$ on the filler concentration is described by the percolation theory and by a scaling law of the form $\sigma=\sigma_{o}\left(\phi-\phi_{c}\right)^{t}$ where $\phi_{c}$ is the percolation threshold and $t$ an exponent depending on the system dimensionality. ${ }^{14}$ The electrical property of the MWCNT-epoxy composites also depends upon many factors i.e. length and alignment of MWCNT, curing of composite, ${ }^{15}$ dispersion of MWCNT in epoxy, etc. In recent decades CNTs have been intensively studied as a promising candidate for epoxy resins at low content. If the filler content is not sufficient for making the percolation network then in that case at the onset of percolation, the charge transport follows a thermal fluctuation induced tunneling mechanism, in which the electrons through the thermally induced fluctuating potential barrier formed by a thin insulating polymer layer separating multiwalled carbon nanotubes (MWCNTs) aggregates.

Researchers nowadays are also working on increasing the thermal conductivity of the polymer nanocomposites. Heat conduction in polymer materials is through elastic vibrations of the lattice. The incorporation of high-thermal conductive fillers enhances the heat transfer rate by forming a conduction path. In case of low filler loading, boundary of fillers and dispersion of fillers are important for the improvement in thermal conductivity. The thermal conductivity of the composites is largely governed by the conductive paths formed by the fillers and reinforcing agents. The thermal conduction behavior of epoxy resin with various fillers was studied and concluded that the epoxy resin produced high-thermal conductive materials with the incorporation of natural graphite powder. $^{16}$ The effects of the MWCNTs, aluminum nitride particles, and their compounds on the microstructure and thermal conductivity of the composites were investigated by Jiao et $a l .{ }^{17}$ The one-dimensional MWCNTs with superb thermal conductivity bridged the AIN particles to form an excellent network, which provides a faster and more effective pathway for phonon transport in the composites.

In general, composites based on chemically functionalized nanotubes were also investigated for the electrical and mechanical properties because functionalization enables a significant improvement in both dispersion and stress-strain transfer. But the chemical functionalization of CNT always not enhances the electrical properties of the composites. The wall damage of the CNT structures during functionalization is the reason behind the decrease in the electrical properties of the composites. ${ }^{18,19} \mathrm{Li}$ et al. reported the slow increase in the conductivity of the chemically functionalized MWCNT/ PVDF composites with increasing MWCNT concentration. ${ }^{20}$ The treatment of CNTs by chemical functionalization and/or ultra-sonication is widely used to increase the dispersion of nanotubes in solvents. A better dispersion of nanotubes in solvents overcomes the problems of nanotube aggregation in polymer composites processed by the solution casting technique. Recently a novel functionalization was studied by grafting carboxyl terminated poly(acrylonitrile-co-butadiene) CTBN on the side-wall of MWCNT to prepare its composite with epoxy matrix. Incorporation of CTBN grafted MWCNTs in epoxy matrix imparted tremendous improvement in mechanical strength as well as fracture toughness when compared with pristine MWCNT/epoxy composites. The mechanism for this improvement in mechanical properties is attributed to the increase in interfacial strength between nanotube and epoxy matrix through chemical bonding. ${ }^{21}$ Some researchers studied the surface coating of CNTs to improve the dielectric constant of the prepared composite samples. $^{22-28}$ Zhang et al. prepared unique cyanate ester composites with high dielectric constant based on multifunctional CNTs coated with phosphaphenanthrene terminated hyperbranched polysiloxane. Qiang et al. ${ }^{29}$ prepared new high dielectric constant composites based on unique multibranched polyaniline and CNT hybrids, in which the multibranched structure, large polyaniline concentration and flexible Si-O chains make the multibranched polyaniline coated on surfaces of CNTs through $\pi-\pi$ interaction and the CNT hybrids have good dispersion in epoxy matrix. Yu et $a{ }^{30}$ synthesized hierarchical composites of polyaniline fibers on the surface of exfoliated graphite by chemical oxidation method, which were used to improve dielectric property of the electroactive polymer poly(vinylidene fluoride).

As a matter of fact, preparing low-cost materials with a high dielectric constant and low dielectric loss based on electric conductors and flexible polymers has attracted worldwide attentions because of the big possibility to inherit the performance advantages of polymers compared with ceramic/polymer composites. ${ }^{31-34}$ Within the past decade, CNTs have gained great attention worldwide owing to their special structure and properties. More interestingly, with the addition of an extremely small content of CNTs to a polymer, the dielectric constant will be greatly increased, suggesting that CNT/polymer composites generally have an extremely low percolation threshold. However, high dielectric constant CNT/polymer composites always exhibit very high dielectric loss, which definitely hinders their application. Moreover, the combination of a large surface area and a high aspect ratio with attractive van der Waal interaction forces in CNTs makes them aggregate to bundles; thus, they are often difficult to mix with polymers. ${ }^{35}$ 
Analyzing the dielectric studies of the MWCNT-epoxy composite filled with low weight percentage of the MWCNT as filler in composite materials at different temperatures and frequencies is the aim of this work. The effect of temperature on the dielectric properties of the prepared specimens at different frequencies is observed. It is reported that the percolation network of the MWCNT in epoxy is formed at 1 weight percentage of the filler. The 1 weight percentage and 4 weight percentage composites are conductor with conductivity as high as respectively $10^{-3}$ and $6 \times 10^{-2} \mathrm{~S} / \mathrm{cm}$. From the electrical properties measurement, the threshold of a conducting or interconnected network of the CNTs was reached at 1 weight percentage. ${ }^{36}$ The dielectric properties of the prepared sample here cannot be described on the basis of the percolation network. Therefore in this work we have chosen to focus our attention on the physical origins of the differences in the electrical conductivity and on the possibility to estimate some material characteristics of the systems under analysis.

\section{Experimental}

\subsection{Materials}

Epoxy resin contains one or more epoxide groups that serve as cross-linking points when the resin reacts with the hardener to form long chains, the polymerization. The hardener has an impact on the matrix structure and the cross linking ratio and by this way the molecular motions.

The thermosetting matrix used in this study was a room temperature cured epoxy resin, provided by M/s Atul Pvt. Ltd. Valsad, India. Figures 1(a)-1(b) show the structure of unmodified epoxy pre-polymer resin and structure of a hardener. The density of the resin cured at room temperature was $1.15 \mathrm{~g} / \mathrm{cm}^{3}$. Industrial grade MWCNT $(1205 \mathrm{YJ})$ was purchased by Nanostructured \& Amorphous Materials, Inc., USA which has Purity more than $95 \%$, outside diameter 10 $20 \mathrm{~nm}$, inside diameter 5-10 nm, length $10-30 \mu \mathrm{m}$, specific surface area $180-230 \mathrm{~m}^{2} / \mathrm{g}$, bulk density $0.04-0.05 \mathrm{~g} / \mathrm{cm}^{3}$.

\subsection{Composite preparation}

Composites filled with different weight percentage $(0.1,0.3$, and 0.5 ) of MWCNT were prepared by the solution casting process. MWCNT was dispersed in the epoxy resin heated at $60^{\circ} \mathrm{C}$ for $30 \mathrm{~min}$ in an ultrasonic bath sonicator without any chemical functionalization or surfactant, then reheated the resin/MWCNT mixture at the same temperature and time for reducing the viscosity and kept in a sonicator for another $30 \mathrm{~min}$. Increasing sonication time may damage the MWCNT. For the homogeneous dispersion of MWCNT in resin, the MWCNT/epoxy mixture was stirred by using a lab stirrer at $200 \mathrm{rpm}$ for $60 \mathrm{~min}$. The hardener is then added in the dispersed MWCNT-epoxy mixture at room temperature. This mixture was cured at $110^{\circ} \mathrm{C}$ for $2 \mathrm{~h}$. The resin/hardener ratio in this preparation was kept at 10:1.

\subsection{Test sample preparation}

Specimens having a different weight percentage of MWCNT filled epoxy composites were prepared. The test specimens were cut from the sheets in the sizes of $1 \mathrm{~mm}$ thickness and $10 \mathrm{~mm}$ diameter. Uniformity of surface was obtained by polishing the specimens. Both sides of the specimens were coated by using air drying type silver conducting paint in such a manner that both the surfaces should not connect electrically with each other. The test specimens were then annealed at $60^{\circ} \mathrm{C}$ for $10 \mathrm{~min}$ and then kept in between the electrodes of the sample holder for various measurements.

\section{Characterization}

\subsection{Dielectric measurements}

The dielectric properties of materials play a key role in the practical performances of integrated circuits. A basic understanding of dielectric properties is therefore needed for engineers and scientists working in semiconductor industries. One important property of dielectric materials is the dielectric constant (permittivity). Dielectric constant $\left(\varepsilon^{\prime}\right)$ is a measure of the ability of a material to be polarized by an electric field, and closely related to the capacitance $(C)$ i.e., the ability to store electric charge. Capacitance $(C)$ and $\tan \delta$ values were measured by using a Wayne Kerr 6500B Impedance Analyzer in the temperature range from $30^{\circ} \mathrm{C}$ to $185^{\circ} \mathrm{C}$ at different frequencies $(0.25 \mathrm{kHz}$ to $5 \mathrm{kHz})$ keeping the heating rate constant at $2^{\circ} \mathrm{C} / \mathrm{min}$. Dielectric constant $\left(\varepsilon^{\prime}\right)$ of the composite has been calculated by using the following relation

$$
\varepsilon^{\prime}=\frac{C}{C_{O}}
$$

where $C$ and $C_{O}$ are the capacitance with and without dielectric, respectively; $C_{O}$ in $p f$ is given by

$$
C_{O}=\frac{(0.08854) A}{d} p f \text {, }
$$

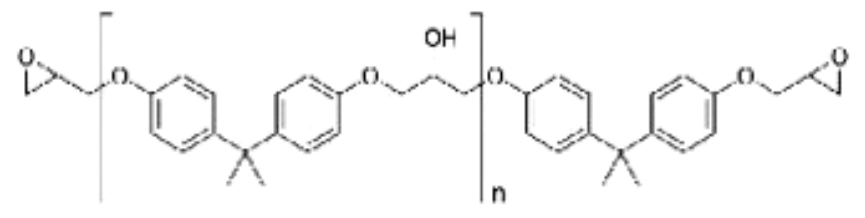

(a)

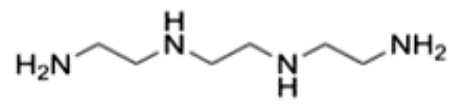

(b)

Fig. 1. (a) Structure of unmodified epoxy pre-polymer resin. (b) Structure of a hardener. 
where $A\left(\mathrm{~cm}^{2}\right)$ is the area of the electrodes and $d(\mathrm{~cm})$ the thickness of the sample.

Dielectric dissipation factor $(\tan \delta)$ is defined as follows

$$
\tan \delta=\frac{\varepsilon^{\prime \prime}}{\varepsilon^{\prime}},
$$

where $\varepsilon^{\prime \prime}$ is the dielectric loss.

In dielectric analysis, the sample is placed between two parallel electrodes. By applying a sinusoidal voltage, an alternating electric field is created, due to which polarization is produced in the sample, which oscillates at the same frequency as the electric field, but has a phase angle shift. The phase angle shift is measured by comparing the applied voltage to the measured current, which is separated into capacitive and conductive components. ${ }^{37,38}$ Measurements of capacitance and conductance are used to calculate:

(i) Real part of permittivity (apparent permittivity) $\varepsilon^{\prime}$, which is proportional to the capacitance and measures the alignment of dipoles.

(ii) Imaginary part of permittivity (loss factor) $\varepsilon^{\prime \prime}$, which is proportional to the conductance and represents the energy required to align dipoles and move ions.

(iii) Dissipation factor, $\tan \delta=\varepsilon^{\prime \prime} / \varepsilon^{\prime}$.

AC conductivity $\left(\sigma_{\mathrm{ac}}\right)$ was calculated from the relation

$$
\sigma_{\mathrm{ac}}=\varepsilon_{0} \omega \varepsilon^{\prime} \tan \delta,
$$

where $\varepsilon_{0}$ is the permittivity of free space, $\tan \delta$ the dielectric dissipation factor and $\omega$ the angular frequency.

At lower and intermediate frequencies $\varepsilon^{\prime}$ and $\tan \delta$ values in MWCNT reinforced epoxy composites are due to the contributions of orientation, space charge and interfacial polarization. Contribution of orientation polarization decreases at high frequency because the molecules do not have time for orientation which is evidenced by the decrease in $\varepsilon^{\prime}$ and $\tan \delta$ of composites with frequency.

\subsection{Scanning electron microscope analysis}

Scanning Electron Microscope (SEM) images of the prepared samples were taken by JEOL JSM 6390A at different magnifications. The prepared samples were coated with gold in a vacuum coating unit prior to the examination.

\section{Results and Discussions}

\subsection{Dielectric constant $\left(\varepsilon^{\prime}\right)$ study}

Figures 2(a)-2(d) show the variation of dielectric constant $\left(\varepsilon^{\prime}\right)$ with temperature $(T)$ for pure epoxy (EP-00), 0.1 weight percentage MWCNT (EP CNT 0.1), 0.3 weight percentage MWCNT (EP CNT 0.3) and 0.5 weight percentage MWCNT (EP CNT 0.5) composites at $0.25,1,2,3$ and $5 \mathrm{kHz}$, respectively. It can be ascertained that the dielectric constant $\left(\varepsilon^{\prime}\right)$ of composites is closely related to the frequency, Fig. 2(a) shows that dielectric constant $\left(\varepsilon^{\prime}\right)$ increases with increase of temperature from $30^{\circ} \mathrm{C}$ to $185^{\circ} \mathrm{C}$ and decreases with an increase in frequency from $0.25 \mathrm{kHz}$ to $5 \mathrm{kHz}$. At low frequencies, all the dipole groups in the epoxy molecular chains can orient themselves, resulting in higher dielectric constant. When the frequency of ac voltage increases, the polarization fails to settle itself completely and the values of the dielectric constant of epoxy resin begin to drop at very high frequencies. The peak height of the transition temperature $\left(T_{t}\right)$ decreases with increasing frequency. At lower temperatures $\varepsilon^{\prime}$ values at different frequencies merged. Figure 2(b) shows that dielectric constant increase with increase in temperature and decreases with the increase of frequency from $0.25 \mathrm{kHz}$ to $5 \mathrm{kHz}$. It is observed that the $\varepsilon^{\prime}$ increases initially with temperature up to $100^{\circ} \mathrm{C}$ after that it decreases up to $150^{\circ} \mathrm{C}$ and again increases up to $185^{\circ} \mathrm{C}$. Figure 2(c) shows that the dielectric constant $\left(\varepsilon^{\prime}\right)$ increases initially with temperature up to $100^{\circ} \mathrm{C}$ and then decreases with temperature until it increased at $150^{\circ} \mathrm{C}$. The same trend can be seen in Fig. 2(d) with the difference that in this curve the value of the dielectric constant $\left(\varepsilon^{\prime}\right)$ increases up to 14 at $185^{\circ} \mathrm{C}$ for $0.25 \mathrm{kHz}$ frequency. The reason behind the increase in $\varepsilon^{\prime}$ after $150^{\circ} \mathrm{C}$ may be when the temperature is lower than a transition temperature $\left(T_{t}\right)$ of the composite the expansion of the polymer matrix will separate the MWCNT fillers which contributes for the conducting path by the electron tunneling before. As the interfaces between the MWCNTs and the polymer matrix will increase by increasing the temperature, the dielectric constant will increase. When the temperature is higher than $T_{t}$, the epoxy crystalline phase begins to melt, transforming to the rubbery flow region. This will make it easier for the fillers to connect or transfer the electron tunneling with each other. Therefore interfaces between the MWCNTs and the polymer matrix will decrease hence, the dielectric constant will decrease. On the other hand, the electrons will capture more energy when the temperature increases, and they can overcome the potential barrier easily. So the dielectric constant decreases with increasing temperature. However, the nomadic electrons will get larger energy and agglomerated on the interfaces at higher temperatures. The polarization will be enlarged, even exceed the influence of MWCNTs again. The dielectric constant increases again. ${ }^{39}$

There are two mechanisms of electrical conduction. One is when the conductors in composites connect with each other to form conductive routes, and the other is that the conductors in composites do not connect with each other, but their distances are so small that the electrons can be transmitted through electron tunnels formed among conductors nearby. Obtained current in first mechanism is named as a leakage current and in second the resultant current is named as a tunneling current. Generally, being higher the leakage current makes more contribution to conductivity than tunneling current. Hence for the leakage current, the content of filler should be high so that a network is formed in the matrix. Thus, the conduction mechanism of the composites is closely 


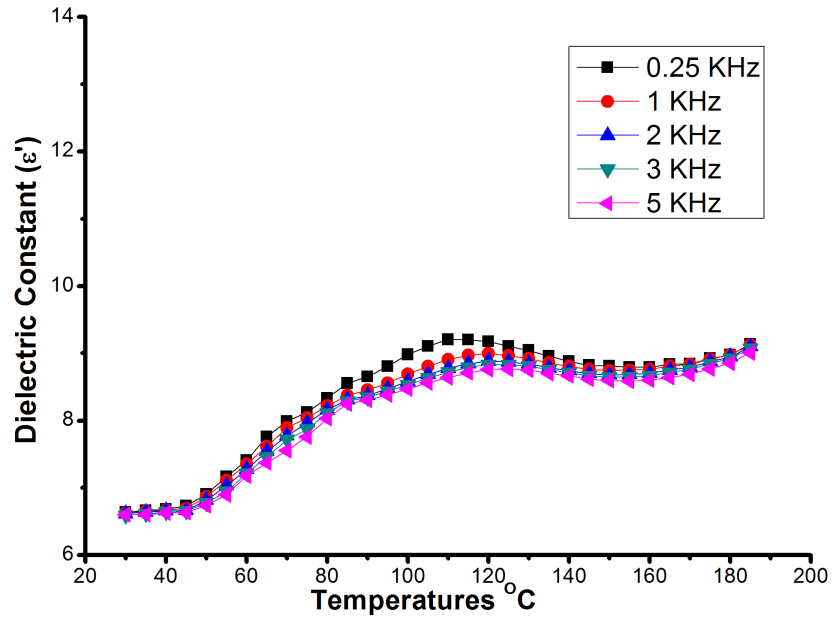

(a)

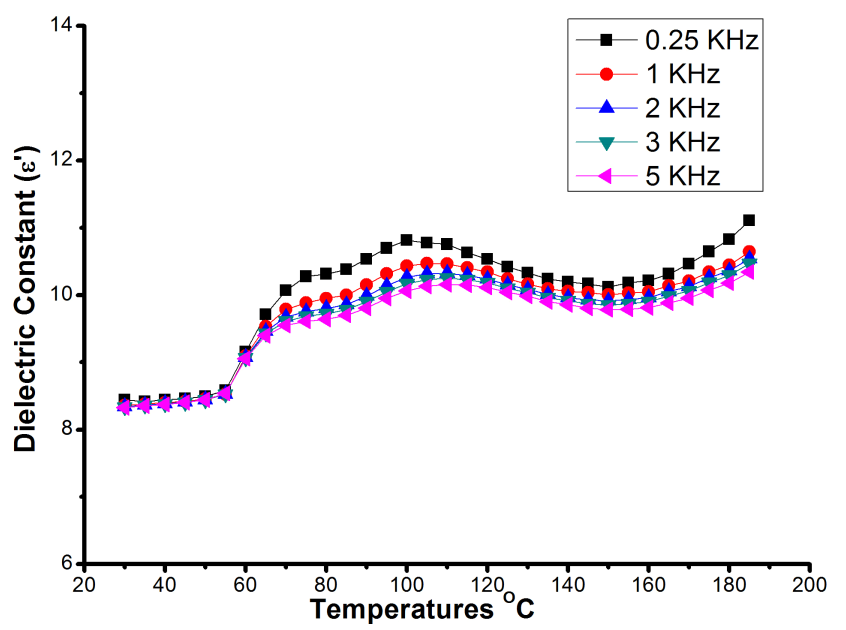

(c)

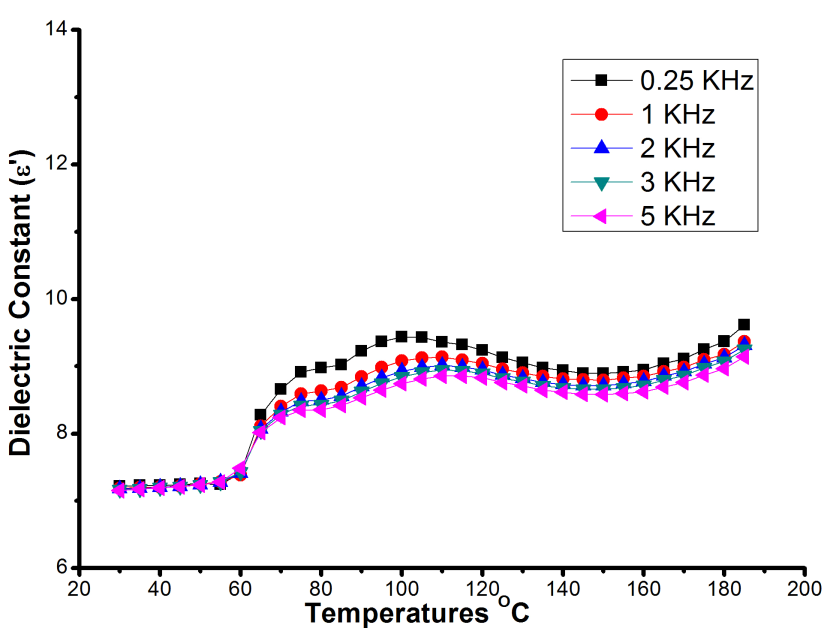

(b)

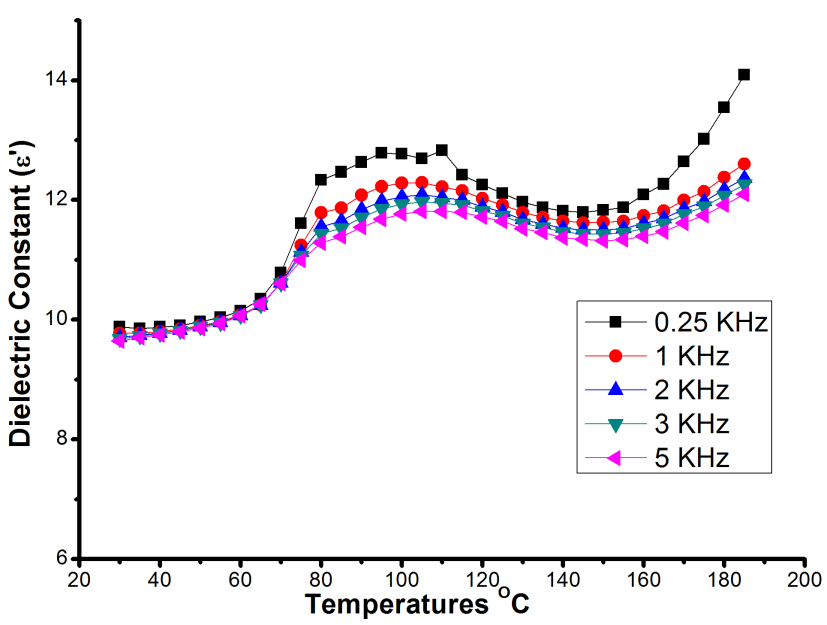

(d)

Fig. 2. The variation of dielectric constant $\left(\varepsilon^{\prime}\right)$ with temperature $(T)$ for (a) pure epoxy (EP-00), (b) 0.1 weight percentage MWCNT (EP CNT 0.1 ), (c) 0.3 weight percentage MWCNT (EP CNT 0.3) and (d) 0.5 weight percentage MWCNT (EP CNT 0.5) composites at $0.25,1,2,3$ and $5 \mathrm{kHz}$, respectively.

related to both the dispersion and the content of conductors. Specifically, when the content of MWCNTs is very small, that is, the content of MWCNTs in the composites is not sufficient to make the connecting networks; hence their conduction mechanisms are due to the tunneling of electron. ${ }^{40}$

\subsection{Dielectric dissipation factor (tan $\delta$ ) analysis}

Figures 3(a)-3(d) show the variation of dissipation factor $(\tan \delta)$ with temperature $(T)$ for EP-00, EP CNT 0.1, EP CNT 0.3 and EP CNT 0.5 composites at $0.25,1,2,3$ and $5 \mathrm{kHz}$, respectively. It is observed that the value of dissipation factor $(\tan \delta)$ increases with the increase in temperature and decreases with an increase in frequency. Dissipation factor $(\tan \delta)$ is the ratio of the electrical power dissipated in a material to the total power circulating in the circuit. In polymers or their composites, $\tan \delta$ is a function of the electrical conductivity (which depends on the charge carrier mobility) and the applied excitation frequency. There are two different interacting processes which can be responsible for the $\tan \delta$ behavior in nanocomposites. The first one is the number of charge carriers which are available for electrical conduction within the composite and the other is the number of interfaces and polymer chain entanglements in the bulk. ${ }^{41}$ It can be ascertained from the plots that in all the four samples, there is a continuous decrease in $\tan \delta$ values with increasing frequency for all filler weight percentage and at lower temperatures the values of $\tan \delta$ are approximately same, there is an increase in the values of $\tan \delta$ after $60^{\circ} \mathrm{C}$ temperature as shown in the inset curves. It is clear from the curves that at lower frequencies the value of $\tan \delta$ is higher than that of the higher frequency. The most probable reason for this observation is a decrease in electrical conductivity in the epoxy composites with increasing frequency which is 


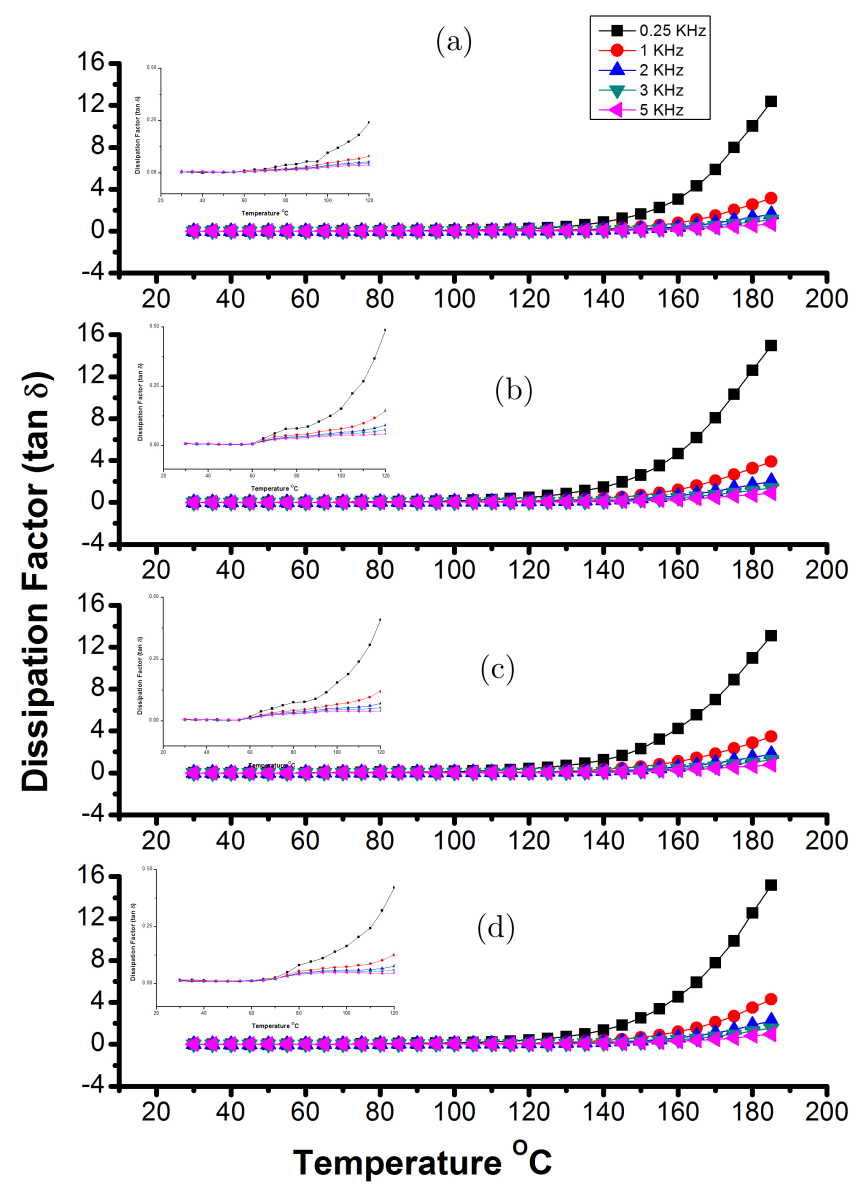

Fig. 3. The variation of dielectric dissipation factor $(\tan \delta)$ with temperature ( $T$ ) for (a) EP-00, (b) EP CNT 0.1, (c) EP CNT 0.3 and (d) EP CNT 0.5 composites at $0.25,1,2,3$ and $5 \mathrm{kHz}$, respectively.

caused by the inability of the charge carriers to traverse the thickness of the material at the higher frequencies. At high frequencies, the motion of charge carriers contributing to the conductivity primarily occurs along polymer chains. ${ }^{40} \mathrm{~A}$ barrier to the charge transport in polymers (causing reduction in electrical conductivity) can occur due to defects, interchain charge transport and transport through interfaces. Probably, in composites, the presence of a large number of interfaces and polymer chain entanglements inhibits the motion of charges in the system, which in turn causes a reduction in the electrical conductivity (hence a lower $\tan \delta$ value).

\subsection{AC conductivity $\left(\sigma_{\mathrm{ac}}\right)$}

Figures 4(a)-4(d) show the plots of AC conductivity $\left(\sigma_{\text {ac }}\right)$ with temperature $(T)$ for EP-00, EP CNT 0.1, EP CNT 0.3 and EP CNT 0.5 composites at $0.25,1,2,3$ and $5 \mathrm{kHz}$, respectively. These plots show that the AC conductivity increased with increasing temperature and frequency. The inset curves clearly show the variation of the $\mathrm{AC}$ conductivity

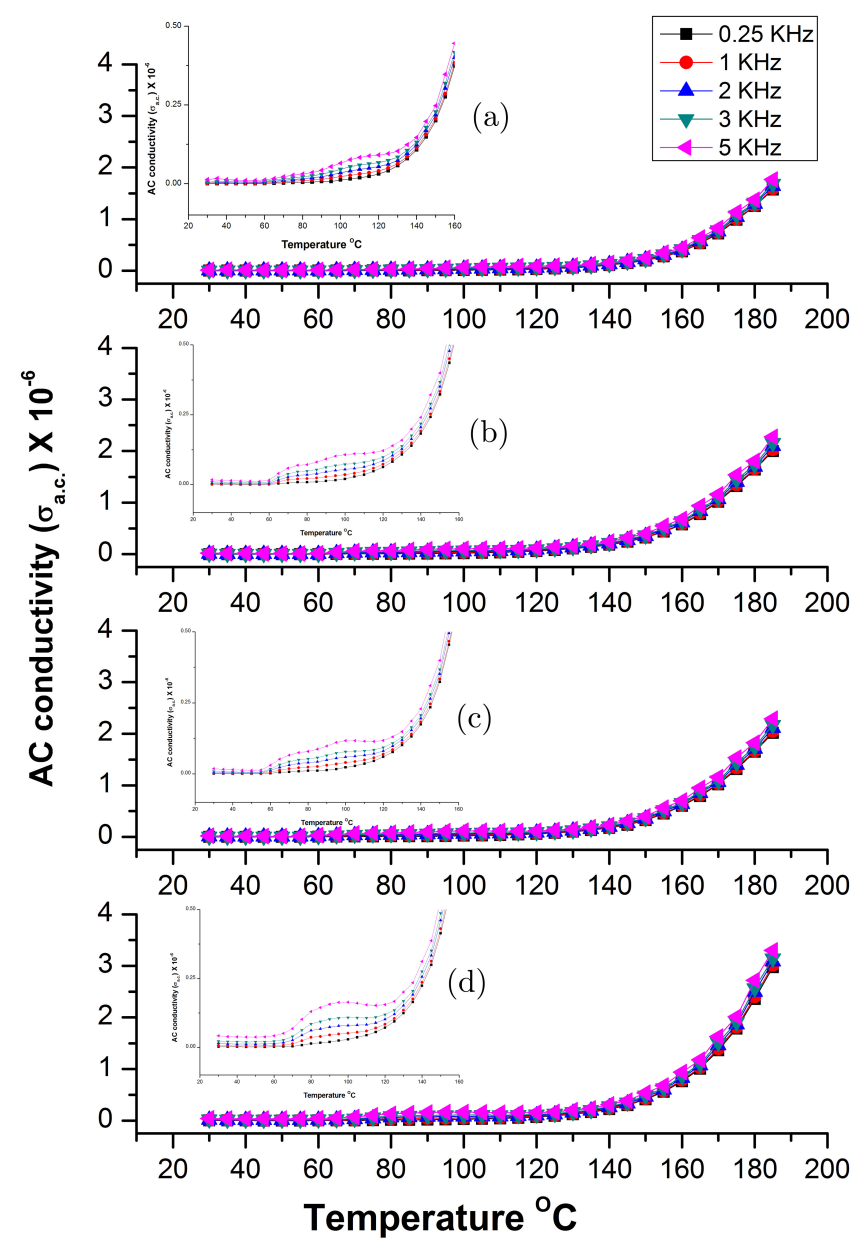

Fig. 4. The plots of AC conductivity $\left(\sigma_{\mathrm{ac}}\right)$ with temperature $(T)$ for (a) EP-00, (b) EP CNT 0.1, (c) EP CNT 0.3 and (d) EP CNT 0.5 composites at $0.25,1,2,3$ and $5 \mathrm{kHz}$, respectively.

with temperature and frequency up to $160^{\circ} \mathrm{C}$. The $\mathrm{AC}$ conductivities for EP CNT 0.1, EP CNT 0.3 and EP CNT 0.5 composites are higher than that of pure epoxy (EP-00) at all frequencies. It is observed that AC conductivity $\left(\sigma_{\mathrm{ac}}\right)$ of all the four samples increases with the increase in temperature and that confirms the negative coefficient of resistance behavior. The AC conductivity of the sample increased with the increase in weight percentage of the MWCNT. This behavior also suggests that the electrical conduction is increasing at the higher temperature which may be again due to the increase in the segmental mobility of the polymer molecules.

\subsection{Dielectric constant, dissipation factor and AC conductivity with $\log f$}

Figures 5(a)-5(c) show the variation of $\varepsilon^{\prime}, \tan \delta$ and $\left(\sigma_{\text {ac }}\right)$ with $\log f$ (frequency) for EP-00, EP CNT 0.1, EP CNT 0.3 and EP CNT 0.5 composites at $100^{\circ} \mathrm{C}$, respectively. It was observed that the $\varepsilon^{\prime}$ and $\tan \delta$ decreased with increasing frequency and ac conductivity increased with increasing 


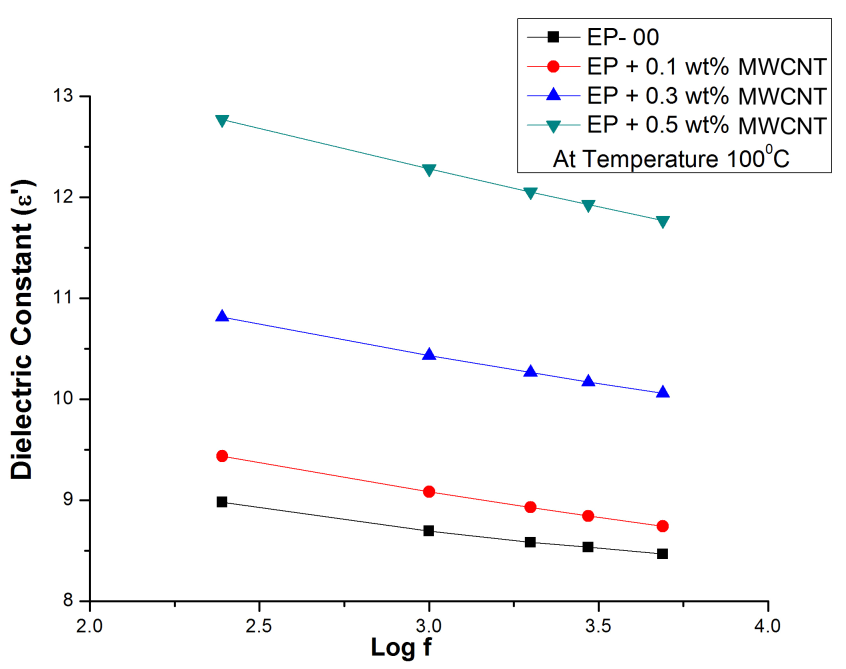

(a)

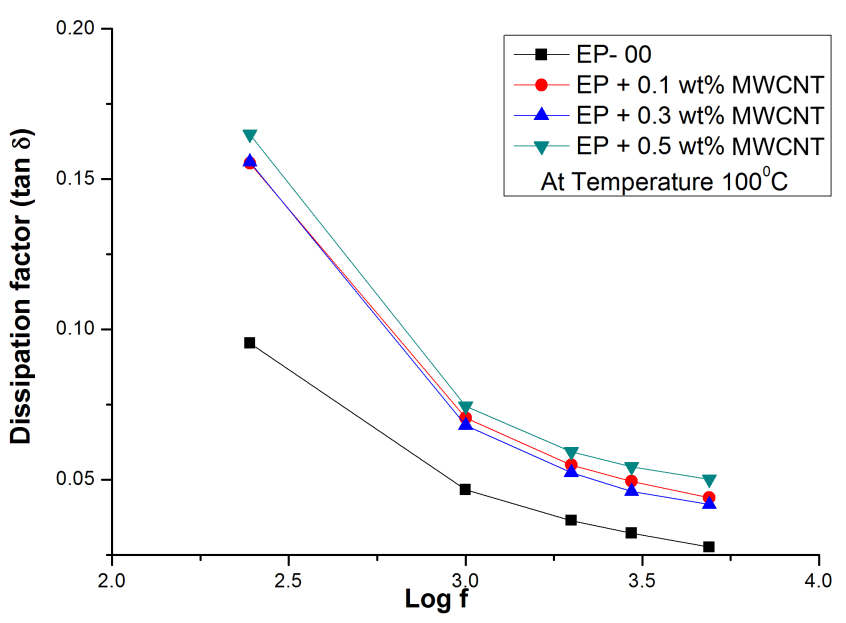

(b)

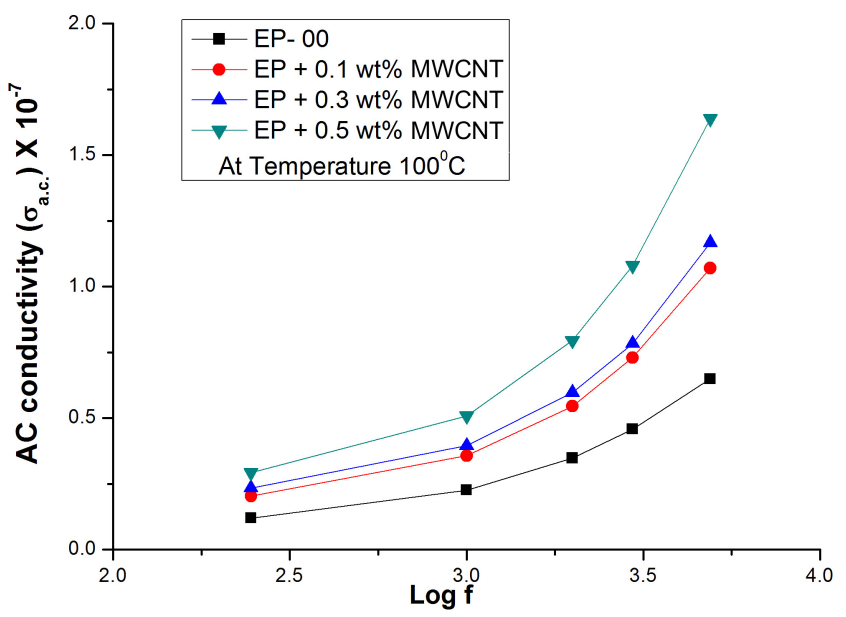

(c)

Fig. 5. The variation of dielectric constant $\left(\varepsilon^{\prime}\right)$, dielectric dissipation factor $(\tan \delta)$ and $\mathrm{AC}$ conductivity $\left(\sigma_{\mathrm{ac}}\right)$ with $\log f$ (frequency) for (a) EP-00, (b) EP CNT 0.1, (c) EP CNT 0.3 and (d) EP CNT 0.5 composites at $100^{\circ} \mathrm{C}$. frequency. The change of $\varepsilon^{\prime}$ and $\tan \delta$ at lower frequency region is higher than that of at high frequency. In AC conductivity an interesting point was observed that the sudden increase seen in the value at $1 \mathrm{kHz}$ frequency for EP CNT 0.5 whereas a gradual increase in the value of EP CNT 0.1 and EP CNT 0.3.

\subsection{Scanning electron microscopy}

Figures 6-9 show the SEM micrographs of a fractured surface of pure epoxy and EP CNT 0.1, EP CNT 0.3 and EP CNT 0.5 composites. It shows the morphology of the fractured surfaces for the neat epoxy and the epoxy/MWCNT nanocomposites used for experiments. In the case of the neat epoxy (Fig. 6), the fractured portions of river line patterns can be observed, indicating brittle fracture and the other micrographs exhibit fracture of tough stuff. In Figs. 7-9, SEM images of the EP CNT 0.1, EP CNT 0.3 and EP CNT 0.5 composites show the dispersion of the MWCNT in an epoxy matrix.

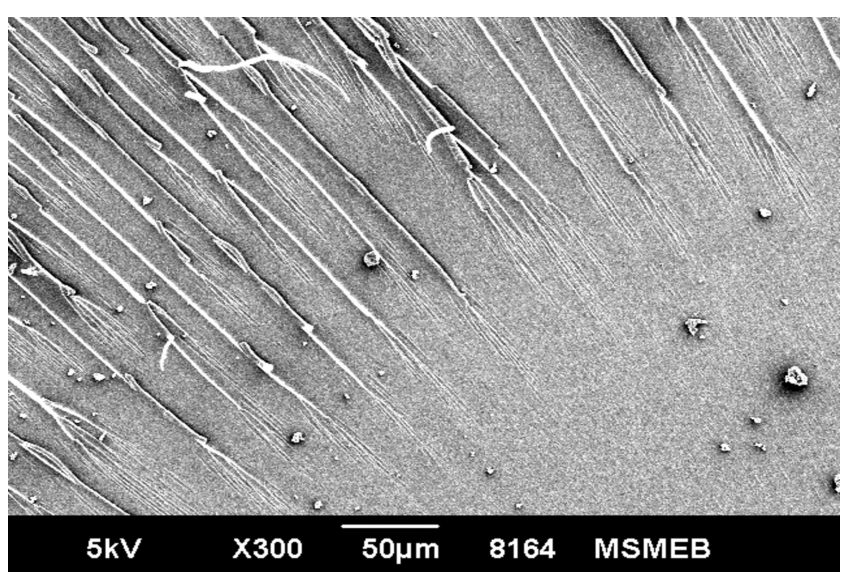

Fig. 6. SEM micrographs of pure epoxy (EP-00).

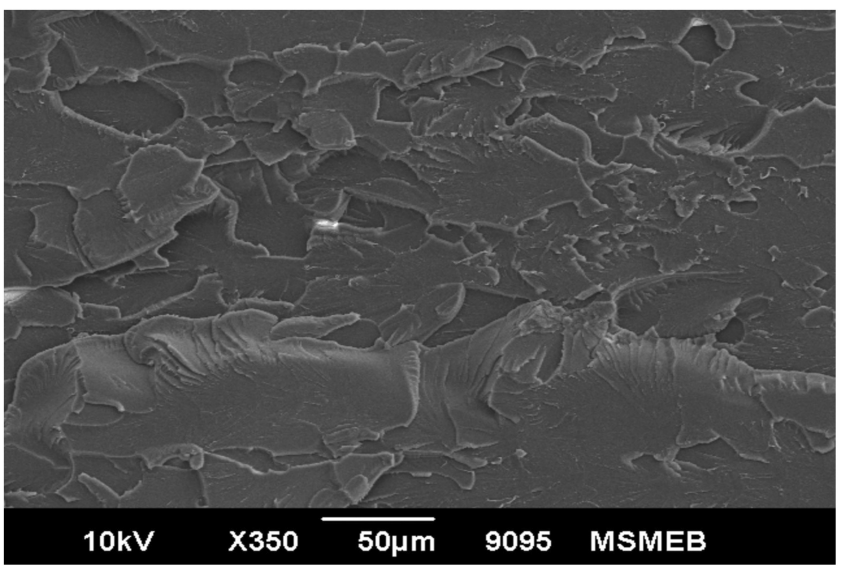

Fig. 7. SEM micrographs of 0.1 weight percentage MWCNT (EP CNT 0.1). 


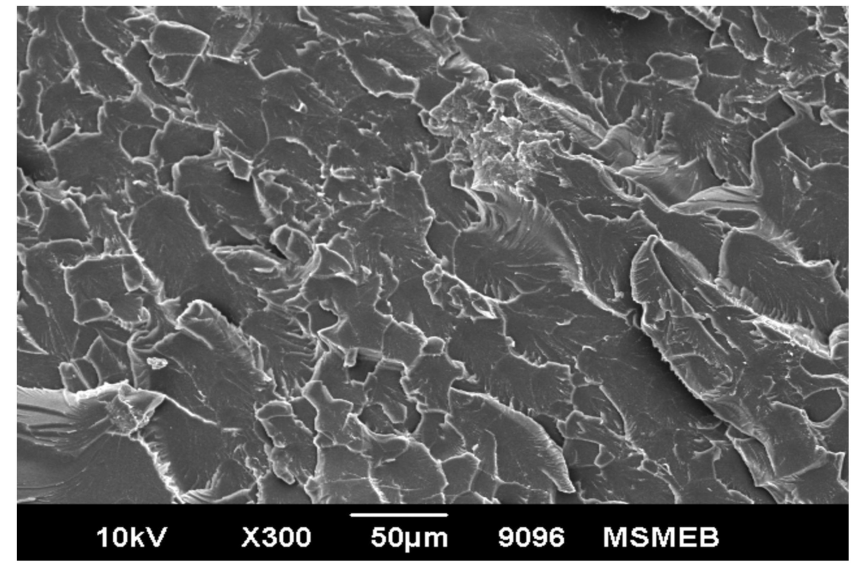

Fig. 8. SEM micrographs of 0.3 weight percentage MWCNT (EP CNT 0.3).

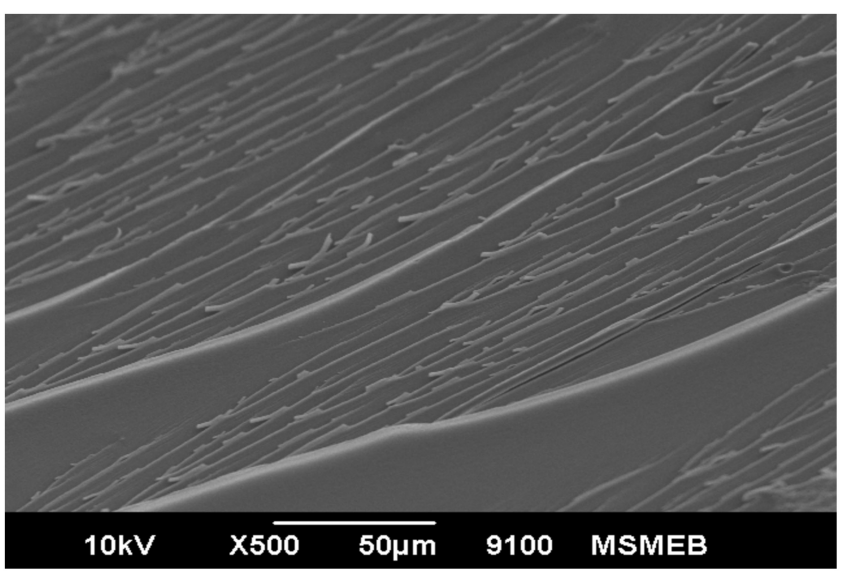

Fig. 9. SEM micrographs of 0.5 weight percentage MWCNT (EP CNT 0.5).

\section{Conclusion}

The small quantity addition of MWCNT can modify considerably the electrical behavior of a MWCNT-epoxy nanocomposite. The conduction mechanism of the polymer nanocomposites is closely related to both the dispersion of the filler as well as the contents of the filler. At low weight percentage of MWCNT in epoxy composites, the conduction was due to the charge transport which follows through a thermal fluctuation induced tunneling mechanism in place of percolation network formation. The addition of 0.5 weight percentage of MWCNT into the epoxy matrix has a remarkable effect on the dielectric constant $\left(\varepsilon^{\prime}\right)$, dissipation factor $(\tan \delta)$ and $\mathrm{AC}$ conductivity $\left(\sigma_{\mathrm{ac}}\right)$ values. In this way the electrical properties of MWCNT-epoxy composites can be enhanced using low weight percentage filler by retaining the mechanical properties of the composites.

\section{Acknowledgments}

Manindra Trihotri owes sincere thanks to Director, Maulana Azad National Institute of Technology, Bhopal 462003 (MP), India for research fellowship and providing laboratory facilities. The support and discussion with the Head of Department Physics (Dr. Rajneesh kurchania) Maulana Azad National Institute of Technology, Bhopal 462051 (MP), India is gratefully acknowledged.

\section{References}

${ }^{1}$ F. Lux, Models proposed to explain the electrical conductivity of mixtures made of conductive and insulating materials, J. Mater. Sci. 28, 285 (1993).

${ }^{2}$ I. Mazov, V. Kuznetsov, A. Romanenko and V. Suslyaev, Properties of MWCNT-Containing Polymer Composite Materials Depending on their Structure, Chapter 3 (InTech, 2012), http://dx. doi.org/10.5772/48245.

${ }^{3}$ J. Wang, H. Kou, X. Liu, Y. Pan and J. Guo, Reinforcement of mullite matrix with multiwalled carbon nanotubes, Ceram. Int. 33, 719 (2007).

${ }^{4}$ Y.-T. Jang, S.-I. Moon, J.-H. Ahn, Y.-H. Lee and B.-K. Ju, A simple approach in fabricating chemical sensor using laterally grown multi-walled carbon nanotubes, Sens. Actuators B 99, 118 (2004).

${ }^{5}$ N. Sinha, J. Ma and J. T. W. Yeow, Carbon nanotube-based sensors, J. Nanosci. Nanotechnol. 6, 573 (2006).

${ }^{6}$ N. Maksimova, G. Mestl and R. Schlogl, Catalytic activity of carbon nanotubes and other carbon materials for oxidative dehydrogenation of ethyl benzene to styrene, Stud. Surf. Sci. Catal. 133, 383 (2001).

${ }^{7}$ M. S. Saha, R. Li and X. Sun, High loading and monodispersed Pt nanoparticles on multiwalled carbon nanotubes for high performance proton exchange membrane fuel cells, J. Power Sources 177, 314 (2008).

${ }^{8}$ M. H. Al-Saleh and U. Sundararaj, Electromagnetic interference shielding mechanisms of CNT/polymer composites, Carbon 47, 1738 (2009).

${ }^{9}$ I. W. Nam, H. K. Lee and J. H. Jang, Electromagnetic interference shielding/absorbing characteristics of CNT-embedded epoxy composites, Composites Part A 42, 1110 (2011).

${ }^{10}$ K.-Y. Park, S.-E. Lee, C.-G. Kim and J.-H. Han, Application of MWNT-added glass fabric/epoxy composites to electromagnetic wave shielding enclosures, Compos. Struct. 81, 401 (2007).

${ }^{11}$ T. A. Hilder and J. M. Hill, Carbon nanotubes as drug delivery nanocapsules, Current Appl. Phys. 8, 258 (2008).

${ }^{12}$ C. Tripisciano, K. Kraemer, A. Taylor and E. Borowiak-Palen, Single-wall carbon nanotubes based anticancer drug delivery system, Chem. Phys. Lett. 478, 200 (2009).

${ }^{13}$ L. Bokobza, Multiwall carbon nanotube elastomeric composites: A review, Polymer 48, 4907 (2007).

${ }^{14} \mathrm{~L}$. Guadagno et al., Effect of functionalization on the thermomechanical and electrical behavior of multi-wall carbon nanotube/ epoxy composites, Carbon 49, 1919 (2011).

${ }^{15}$ M. Trihotri, U. K. Dwivedi, F. H. Khan, M. M. Malik and M. S. Qureshi, Effect of curing on activation energy and dielectric 
properties of carbon black-epoxy composites at different temperatures, J. Non-Cryst. Solids 421, 1 (2015).

${ }^{16}$ M. A. Vadivelu, C. Ramesh Kumar and G. M. Joshi, Polymer composites for thermal management: A review, Compos. Interfaces (2016), doi: 10.1080/09276440.2016.1176853.

${ }^{17}$ J. Jiao, Y. Cui and Y. Xia, Improved thermal conductivity of epoxy composites prepared with a mixed filler of multiwalled carbon nanotubes and aluminum nitride particles, High Perform. Polym. 1, (2016), doi: 10.1177/0954008316644036.

${ }^{18}$ Y. J. Kim, T. S. Shin, H. D. Choi, J. H. Kwon, Y.-C. Chung and H. G. Yoon, Electrical conductivity of chemically modified multiwalled carbon nanotube/epoxy composites, Carbon 43, 23 (2005).

${ }^{19}$ J. G. Park, Q. Cheng, J. Lu, J. Bao, S. Li, Y. Tian, Z. Liang, C. Zhang and B. Wang, Thermal conductivity of MWCNT/epoxy composites: The effects of length, alignment and functionalization, Carbon 50, 2083 (2012).

${ }^{20}$ Q. Li, Q. Xue, L. Hao, X. Gao and Q. Zheng, Large dielectric constant of the chemically functionalized carbon nanotube/polymer composites, Compos. Sci. Technol. 68, 2290 (2008).

${ }^{21} \mathrm{R}$. Konnola and K. Joseph, Effect of side-wall functionalisation of multi-walled carbon nanotube on the thermo-mechanical properties of epoxy composites, RSC Adv. (2016), doi: 10.1039/ C6RA00080K.

${ }^{22}$ G. Viswanathan, N. Chakrapani, H. Yang, B. Wei, H. Chung, K. Cho, C. Y. Ryu and P. M. Ajayan, Single-step in situ synthesis of polymer-grafted single-wall nanotube composites, J. Am. Chem. Soc. 125, 9258 (2003).

${ }^{23}$ J. Zhu, J. Kim, H. Peng, J. L. Margrave, V. N. Khabashesku and E. V. Barrera, Improving the dispersion and integration of singlewalled carbon nanotubes in epoxy composites through functionalization, Nano Lett. 3, 1107 (2003).

${ }^{24}$ G.-X. Chen, H.-S. Kim, B. H. Park and J.-S. Yoon, Controlled functionalization of multiwalled carbon nanotubes with various molecular-weight poly(l-lactic acid), J. Phys. Chem. B 109, 22237 (2005).

${ }^{25}$ R. R. Nayak, A. M. Shanmugharaj and S. H. Ryu, A novel route for polystyrene grafted single-walled carbon nanotubes and their characterization, Macromol. Chem. Phys. 209, 1137 (2008).

${ }^{26}$ X. Gong, J. Liu, S. Baskaran, R. D. Voise and J. S. Young, Surfactant-assisted processing of carbon nanotube/polymer composites, Chem. Mater. 12, 1049 (2000).

${ }^{27}$ H. Peng, P. Reverdy, V. N. Khabashesku and J. L. Margrave, Sidewall functionalization of single-walled carbon nanotubes with organic peroxides, Chem. Commun. 3, 362-363 (2003).

${ }^{28}$ Y. Tang, J. Gou and Y. Hu, Covalent functionalization of carbon nanotubes with polyhedral oligomeric silsequioxane for superhydrophobicity and flame retardancy, Polym. Eng. Sci. 53, 1021 (2013).

${ }^{29}$ Z. Qiang, G. Liang, A. Gu and L. Yuan, The dielectric behavior and origin of high- $k$ composites with very low percolation threshold based on unique multi-branched polyaniline/carbon nanotube hybrids and epoxy resin, Composites Part A 64, 1 (2014).

${ }^{30}$ L. Yu, Y. Zhang, W. Tong, J. Shang, F. Lv, P. K. Chu and W. Guo, Hierarchical composites of conductivity controllable polyaniline layers on the exfoliated graphite for dielectric application, Composites Part A 43, 2039 (2012).

${ }^{31}$ L. Xie, X. Huang, C. Wu and P. Jiang, Core-shell structured poly (methyl methacrylate)/BaTiO 3 nanocomposites prepared by in situ atom transfer radical polymerization: A route to high dielectric constant materials with the inherent low loss of the base polymer, J. Mater. Chem. 21, 5897 (2011).

${ }^{32}$ X. Zhao, A. A. Koos, B. T. T. Chu, C. Johnston, N. Grobert and P. S. Grant, Spray deposited fluoropolymer/multi-walled carbon nanotube composite films with high dielectric permittivity at low percolation threshold, Carbon 47, 561 (2009).

${ }^{33}$ H. Pang, C. Chen, Y.-C. Zhang, P.-G. Ren, D.-X. Yan and Z.-M. Li, The effect of electric field, annealing temperature and filler loading on the percolation threshold of polystyrene containing carbon nanotubes and graphene nanosheets, Carbon 49, 1980 (2011).

${ }^{34}$ J. Chang, G. Liang, A. Gu, S. Cai and L. Yuan, The production of carbon nanotube/epoxy composites with a very high dielectric constant and low dielectric loss by microwave curing, Carbon 50, 689 (2012).

${ }^{35}$ D. Sun, Z. Zhou, G.-X. Chen and Q. Li, Regulated dielectric loss of polymer composites from coating carbon nanotubes with a cross-linked silsesquioxane shell through free-radical polymerization, ACS Appl. Mater. Interfaces 6, 18635 (2014).

${ }^{36}$ A. Allaoui, S. Bai, H. M. Cheng and J. B. Bai, Mechanical and electrical properties of a MWNT/epoxy composite, Compos. Sci. Technol. 62, 1993 (2002).

${ }^{37}$ I. Ben Amora, H. Rekik, H. Kaddami, M. Raihane, M. Arous and A. Kallel, Studies of dielectric relaxation in natural fiber-polymer composites, J. Electrost. 67, 717 (2009).

${ }^{38}$ M. Trihotri, D. Jain, U. K. Dwivedi, F. H. Khan, M. M. Malik and M. S. Qureshi, Effect of silver coating on electrical properties of sisal fibre-epoxy composites, Polym. Bull. 70, 3501 (2013), doi: 10.1007/s00289-013-1036-7.

${ }^{39}$ Q. Li, Q. Z. Xue, X. L. Gao and Q. B. Zheng, Temperature dependence of the electrical properties of the carbon nanotube/ polymer composites, eXPRESS Polymer Lett. 3, 769 (2009).

${ }^{40}$ J. Chang, G. Liang, A. Gu, S. Cai and L. Yuan, The production of carbon nanotube/epoxy composites with a very high dielectric constant and low dielectric loss by microwave curing, Carbon 50, 689 (2012).

${ }^{41}$ S. Singha and M. Joy Thomas, Permittivity and tan delta characteristics of epoxy nanocomposites in the frequency range of $1 \mathrm{MHz}-1 \mathrm{GHz}$, IEEE Trans. Dielectr. Electr. Insul. 15, (2008) 2-11. 